\title{
A Fully Gradient Model for Euler-Bernoulli Nanobeams
}

\author{
Raffaele Barretta, ${ }^{1}$ Raimondo Luciano, ${ }^{2}$ and Francesco Marotti de Sciarra ${ }^{1}$ \\ ${ }^{1}$ Department of Structures for Engineering and Architecture, Via Claudio 25, 80121 Naples, Italy \\ ${ }^{2}$ Department of Civil and Mechanical Engineering, University of Cassino and Southern Lazio, Via G. Di Biasio 43, \\ 03043 Cassino, Italy
}

Correspondence should be addressed to Raffaele Barretta; rabarret@unina.it

Received 1 April 2015; Revised 31 August 2015; Accepted 2 September 2015

Academic Editor: Fumihiro Ashida

Copyright (C) 2015 Raffaele Barretta et al. This is an open access article distributed under the Creative Commons Attribution License, which permits unrestricted use, distribution, and reproduction in any medium, provided the original work is properly cited.

\begin{abstract}
A fully gradient elasticity model for bending of nanobeams is proposed by using a nonlocal thermodynamic approach. As a basic theoretical novelty, the proposed constitutive law is assumed to depend on the axial strain gradient, while existing gradient elasticity formulations for nanobeams contemplate only the derivative of the axial strain with respect to the axis of the structure. Variational equations governing the elastic equilibrium problem of bending of a fully gradient nanobeam and the corresponding differential and boundary conditions are thus provided. Analytical solutions for a nanocantilever are given and the results are compared with those predicted by other theories. As a relevant implication of applicative interest in the research field of nanobeams used in nanoelectromechanical systems (NEMS), it is shown that displacements obtained by the present model are quite different from those predicted by the known gradient elasticity treatments.
\end{abstract}

\section{Introduction}

Nanostructures are nowadays widely used as main components of many micro- and nanoelectromechanical systems (MEMS and NEMS) [1-5]. Typical MEMS and NEMS consist of thin beams made of metals, polymers, traditional siliconbased materials, or functionally graded materials [6-11].

The mechanical behaviour of nanostructures can be studied by both theoretical and experimental methods; see, for example, [12] for a review. Generally, conducting controlled experiments at the nanoscale is difficult and expensive. Accordingly, theoretical modelling is the main tool for studying the properties of nanostructures.

Nanobeams can be modelled by using an atomistic approach $[13,14]$ or continuum mechanics-based models which are extensively used due to computational simplicity and effectiveness. One of the basic assumptions in continuum models is that the lattice structure is neglected and it is replaced by a continuum medium.

In this framework, several theories have been introduced to consider small-scale effects at the micro- and nanoscale.
Unlike classical (local) elastic models, nonlocal elastic models introduce length-scale parameters in the constitutive relations. On the basis of an analogy presented in $[15,16]$, nonlocal effects on nanorods and nanobeams, formulated according to the Eringen model, can be simulated by prescribing suitable axial and curvature distortions on corresponding local rods and beams. Accordingly a general procedure is provided to establish if nonlocal nanorods and nanobeams are free of small-scale effects.

Several models based on the Euler-Bernoulli or Timoshenko beam theories have been proposed in the literature in order to develop effective nonlocal nanobeam models for bending, buckling, and vibrations; see, for example, nonlocal elasticity model [17-24] couple stress theory [25, 26], modified couple stress theory [27-29], gradient plasticity for strain softening materials [30-33], gradient elasticity model [34-40], and FE analysis of finite random composite bodies $[41,42]$.

In particular, the existing gradient elasticity model (GM) for Euler-Bernoulli nanobeams encompasses the first derivative of the axial strain in the beam axis direction (see, e.g., 
the recent contribution by Aifantis and coworkers [40, 43]) and disregards the other nonvanishing component of the axial strain gradient.

The aim of this paper is to propose a fully gradient elasticity model (FUGM) for Euler-Bernoulli nanobeams, starting from a thermodynamic approach, in which all the components of the gradient of the axial strain are taken into account to model the nonlocal behaviour of the nanobeam. The solutions of the FUGM in terms of transverse displacements can thus be compared with the corresponding ones evaluated by the GM. As a result, the influence of the gradient component, which is usually disregarded in the GM, on the bending behaviour of nanobeams can be clearly enlightened.

The starting point of the present work is the definition of the Helmholtz free energy in terms of the axial strain and of its gradient. Accordingly a fully gradient elasticity model (FUGM) for bending of Euler-Bernoulli nanobeams is provided based on a nonlocal thermodynamic approach; see, for example, [44, 45]. Two length-scale parameters are introduced in the model and are associated with the nonvanishing components of the strain gradient of the axial strain.

Nonlocal thermodynamics allows us to build up a reliable methodology to derive the nonlocal variational formulation of the elastic equilibrium for the FUGM which yields the differential equation with the relevant boundary conditions.

As an example, a nanocantilever with a concentrated load at its tip is investigated using the FUGM. Results given by the FUGM are compared with those evaluated by the GM and it is shown that the effects of the gradient component are usually disregarded in the GM; that is, the derivative of the axial strain in the transverse direction is significant. The difference between the results of the FUGM and the results of the GM will be quantitatively shown and analysed. In particular, the nanobeam solved by the FUGM becomes stiffer than the one solved by the GM.

\section{Nonlocal Thermodynamics for Euler-Bernoulli Nanobeams}

The internal energy density $e$ in a nonlocal elastic material can be assumed in the form

$$
e=e(\boldsymbol{\varepsilon}, \nabla \boldsymbol{\varepsilon}, s)
$$

where the kinematic internal variable $\nabla \boldsymbol{\varepsilon}$ is the gradient of the strain tensor $\varepsilon$ and $s$ is the entropy.

Denoting by $\psi(\boldsymbol{\varepsilon}, \nabla \boldsymbol{\varepsilon}, T)$ the Helmholtz free energy defined by means of the Legendre transform, the first law of thermodynamics for isothermal processes and for a nonlocal behaviour (see, e.g., $[44,46])$ can be formulated as follows:

$$
\int_{\mathscr{B}} \dot{\psi}(\boldsymbol{\varepsilon}, \nabla \boldsymbol{\varepsilon}, T) d V=\int_{\mathscr{B}} \boldsymbol{\sigma} * \dot{\boldsymbol{\varepsilon}} d V-\int_{\mathscr{B}} \dot{s} T d V,
$$

where $\dot{T}=0$ and $\sigma$ is the nonlocal stress tensor field of the body $\mathscr{B}$. The superscript dot indicates the differentiation with respect to time and the symbol $*$ denotes the single (double) index saturation. The energy balance in (2) can be written pointwise in $\mathscr{B}$ in the form

$$
\dot{\psi}=\boldsymbol{\sigma} * \dot{\boldsymbol{\varepsilon}}-\dot{s} T+P,
$$

where the thermodynamic scalar variable $P$ is the nonlocal residual function which accounts for the energy exchanges between neighbour particles (see, e.g., [47]). Since nonlocal effects due to elastic deformations are confined into the body, the residual $P$ fulfils the insulation condition:

$$
\int_{\mathscr{B}} P d V=0
$$

The second principle of nonlocal thermodynamics for isothermal processes is written in the local form $\dot{s} T \geq 0$ everywhere in $\mathscr{B}$, where $\dot{s}$ is the internal entropy production rate per unit volume; see, for example, [48]. Accordingly the nonlocal Clausius-Duhem inequality for isothermal processes follows from relation (3) in the form

$$
D=\dot{s} T=\boldsymbol{\sigma} * \dot{\boldsymbol{\varepsilon}}-\dot{\psi}+P \geq 0,
$$

where the presence of the nonlocal residual function $P$ guarantees the nonnegativeness of the dissipation $D$ and accounts for material nonlocality.

The body energy dissipation $\mathscr{E}$ is provided by integrating relation (5) to get

$$
\mathscr{E}=\int_{\mathscr{B}} \dot{\boldsymbol{s}} T d V=\int_{\mathscr{B}} \boldsymbol{\sigma} * \dot{\boldsymbol{\varepsilon}} d V-\int_{\mathscr{B}} \dot{\psi} d V \geq 0 .
$$

Expanding relation (6), dissipation (5) is pointwise vanishing according to the reversible nature of the model. As a consequence, for any admissible deformation mechanism, it results in

$$
\int_{\mathscr{B}} \boldsymbol{\sigma} * \dot{\boldsymbol{\varepsilon}} d V=\int_{\mathscr{B}} d_{\boldsymbol{\varepsilon}} \psi * \dot{\boldsymbol{\varepsilon}} d V+\int_{\mathscr{B}} d_{\nabla \boldsymbol{\varepsilon}} \psi * \nabla \dot{\boldsymbol{\varepsilon}} d V
$$

In the next section, a fully gradient elasticity theory for Euler-Bernoulli nanobeams is presented starting from (7) and taking into account all the components of the strain gradient.

\section{Fully Gradient Elasticity Model for Nanobeams}

Let us consider a homogeneous isotropic nanobeam of length $L$. The $x$-coordinate is taken along the length of the beam and the $y$-coordinate along the thickness and the $z$-coordinate is taken along the width of the beam. The geometry and the applied loads of the nanobeam are such that the displacements $\left(s_{x}, s_{y}, s_{z}\right)$ along the axes $(x, y, z)$ are functions of the $x$ - and $y$-coordinates. It is further assumed that the displacement $s_{z}$ is identically zero. The cross-sectional area $A$ and the second moment of area $I$ about the $z$-axis are

$$
(A, I)=\int_{\Omega}\left(1, y^{2}\right) d A .
$$


The proposed fully gradient elasticity model (FUGM) for Euler-Bernoulli nanobeams is based on the following classical displacement field:

$$
\begin{aligned}
& s_{x}(x, y)=-v^{(1)}(x) y, \\
& s_{y}(x, y)=v(x), \\
& s_{z}(x, y)=0,
\end{aligned}
$$

where $v$ is the transverse displacement and the apex ${ }^{(n)}$ denotes the $n$-derivative of the function - along the nanobeam axis $x$. The rotation $\varphi$ of the cross-section of the nanobeam is $\varphi(x)=v^{(1)}(x)$.

Accordingly the nonvanishing kinematically compatible deformation is given by the axial strain

$$
\varepsilon_{x}(x, y)=-v^{(2)}(x) y
$$

and the related nonvanishing components of the strain gradient are

$$
\begin{aligned}
& \frac{\partial \varepsilon_{x}(x, y)}{\partial x}=-v^{(3)}(x) y, \\
& \frac{\partial \varepsilon_{x}(x, y)}{\partial y}=-v^{(2)}(x)=-\chi
\end{aligned}
$$

where $\chi$ denotes the bending curvature of the nanobeam. In the sequel, for simplicity, the subscript $x$ of strains will be dropped.

3.1. Variational Formulation of Elastic Equilibrium. The proposed FUGM for Euler-Bernoulli nanobeams is governed by the following expression of the free energy:

$$
\psi\left(\varepsilon, \varepsilon^{(1)}\right)=\frac{1}{2} E \varepsilon^{2}+\frac{1}{2} E c_{x}^{2} \varepsilon^{(1)^{2}}+\frac{1}{2} E c_{y}^{2} \chi^{2}(\varepsilon),
$$

where $E$ is Young's modulus. The coefficients $c_{x}=e_{0} l_{x}$ and $c_{y}=e_{0} l_{y}$ incorporate small-scale effects, where $e_{0}$ is a material constant and $\left(l_{x}, l_{y}\right)$ are the material length-scales associated with the variation of the normal strain in the longitudinal direction $x$ and in the transversal direction $y$. Accordingly, the nonlocal model depends on two parameters $c_{x}$ and $c_{y}$.

Note that the nonlocal model tends to the local model in the limit of vanishing nonlocal parameters as shown in the sequel.

Using expression (12) of the free energy, the thermodynamic requirement (7) provides the variational formulation:

$$
\int_{\mathscr{B}} \sigma \dot{\varepsilon} d V=\int_{\mathscr{B}} \sum \dot{\varepsilon} d V+\int_{\mathscr{B}} \sigma_{1} \dot{\varepsilon}^{(1)} d V,
$$

where the static variables $\Sigma$ and $\sigma_{1}$ denote the axial stresses which are, respectively, duals of the strain $\varepsilon$ and of the strain derivative $\varepsilon^{(1)}$. Thus, the axial stresses $\Sigma$ and $\sigma_{1}$ are explicitly given by

$$
\begin{gathered}
\Sigma=\frac{\partial \psi}{\partial \varepsilon}=\sigma_{0}+\sigma_{2}, \\
\sigma_{1}=\frac{\partial \psi}{\partial \varepsilon^{(1)}}=E c_{x}^{2} \varepsilon^{(1)},
\end{gathered}
$$

where $\sigma_{0}=E \varepsilon$ and $\sigma_{2}=E c_{y}^{2} \varepsilon / y^{2}$.

\section{Bending Solution}

The explicit expression of the variational formulation for the FUGM can be recovered by (13). In fact substituting the kinematically compatible relation (10) and the expression of the strain derivatives (11) in (13) we get the nonlocal variational formulation associated with the FUGM:

$$
\begin{aligned}
\int_{0}^{L} M \dot{v}^{(2)} d x= & \int_{0}^{L} M_{0} \dot{v}^{(2)} d x+\int_{0}^{L} M_{1} \dot{v}^{(3)} d x \\
& +\int_{0}^{L} B \dot{v}^{(2)} d x,
\end{aligned}
$$

where the stress resultant moments are

$$
\left(M, M_{0}, M_{1}, B\right)=-\int_{\Omega}\left(\sigma, \sigma_{0}, \sigma_{1}, \sigma_{2}\right) y d A .
$$

The differential equilibrium equation and the boundary conditions corresponding to the proposed nonlocal FUGM can be obtained by applying the integration by parts to the nonlocal variational formulation (15) to get

$$
\dot{v}: M^{(2)}=M_{0}^{(2)}-M_{1}^{(3)}+B^{(2)}
$$

and the boundary conditions are

$$
\begin{aligned}
& \text { specify } v \text { or }-M^{(1)}=-M_{0}^{(1)}+M_{1}^{(2)}-B^{(1)}, \\
& \text { specify } v^{(1)} \text { or } M=M_{0}-M_{1}^{(1)}+B, \\
& \text { specify } v^{(2)} \text { or } 0=M_{1} .
\end{aligned}
$$

The classical differential equilibrium relation can be recovered by integrating by parts the l.h.s. of (15) to get $M^{(2)}=p_{y}$, where $p_{y}$ is the distributed transversal load. The boundary conditions at $x=\{0, L\}$ provide the relations $T=-M^{(1)}=\mathscr{F}$ and $M=\mathscr{M}$, where $T$ is the shear force and $(\mathscr{F}, \mathscr{M})$ are the transverse force and couple, respectively.

Differential equation (17) and boundary conditions (18) of the FUGM can be reformulated in terms of the transverse displacement $v$ by expressing the bending moments $\left(M_{0}, M_{1}, B\right)$ in the following forms:

$$
\begin{aligned}
\left(M_{0}, M_{1}\right) & =-\int_{\Omega} E y\left(\varepsilon, c_{x}^{2} \varepsilon^{(1)}\right) d A=E I\left(v^{(2)}, c_{x}^{2} v^{(3)}\right), \\
B & =-\int_{\Omega} E c_{y}^{2} \frac{\varepsilon}{y} d A=E A c_{y}^{2} v^{(2)}
\end{aligned}
$$

so that the differential equilibrium equation for the nonlocal FUGM can be obtained by substituting (19) into (17) and (18) to get

$$
\dot{v}: E\left(I+c_{y}^{2} A\right) v^{(4)}-c_{x}^{2} E I v^{(6)}=p_{y}
$$

and the related boundary conditions are

$$
\begin{aligned}
& \text { specify } v \text { or } T=-E\left(I+c_{y}^{2} A\right) v^{(3)}+c_{x}^{2} E I v^{(5)} \text {, } \\
& \text { specify } v^{(1)} \text { or } M=E\left(I+c_{y}^{2} A\right) v^{(2)}-c_{x}^{2} E I v^{(4)} \text {, } \\
& \text { specify } v^{(2)} \text { or } 0=E I c_{x}^{2} v^{(3)} .
\end{aligned}
$$


The bending moment can then be obtained in terms of the transverse displacement by performing an integration by parts of the second term at the r.h.s. of (15). Hence, we have

$$
M=M_{0}-M_{1}^{(1)}+B=E\left(I+c_{y}^{2} A\right) v^{(2)}-c_{x}^{2} E I v^{(4)}
$$

and the boundary condition $M_{1}=0$ at $x=\{0, L\}$ is fulfilled due to $(18)_{3}$.

The boundary conditions to be imposed at the end of a nanobeam to determine the six unknown coefficients associated with the FUGM are reported hereafter for external constraints of engineering interest:

(i) simply supported end at the point $x=x_{0}$

$$
\begin{aligned}
v\left(x_{0}\right) & =0, \\
E\left(I+c_{y}^{2} A\right) v^{(2)}\left(x_{0}\right)-c_{x}^{2} E I v^{(4)}\left(x_{0}\right) & =0, \\
v^{(3)}\left(x_{0}\right) & =0 ;
\end{aligned}
$$

(ii) clamped end at the point $x=x_{0}$

$$
\begin{aligned}
v\left(x_{0}\right) & =0, \\
v^{(1)}\left(x_{0}\right) & =0, \\
v^{(3)}\left(x_{0}\right) & =0 ;
\end{aligned}
$$

(iii) free end at the point $x=x_{0}$ loaded by a couple $\mathscr{M}$ and a force $\mathscr{F}$ :

$$
\begin{aligned}
-E\left(I+c_{y}^{2} A\right) v^{(3)}\left(x_{0}\right)+c_{x}^{2} E I v^{(5)}\left(x_{0}\right) & =\mathscr{F}, \\
E\left(I+c_{y}^{2} A\right) v^{(2)}\left(x_{0}\right)-c_{x}^{2} E I v^{(4)}\left(x_{0}\right) & =\mathscr{M}, \\
v^{(3)}\left(x_{0}\right) & =0 .
\end{aligned}
$$

Remark 1. If the parameter $c_{y}$ is vanishing, the governing equation (20) and the boundary conditions (21) pertaining to the FUGM degenerate to the corresponding relations of the nonlocal Euler-Bernoulli nanobeam theory based on the gradient elasticity model (GM). Moreover, if both the material parameters $c_{x}$ and $c_{y}$ are equal to zero, the governing equations (20) and boundary conditions (21) reduce to those of the classical (local) Euler-Bernoulli beam model.

\section{Example: Cantilever Nanobeam}

The solutions of the FUGM obtained in the previous sections are specialized for a nanocantilever subjected to a concentrated load $F$ at its tip in terms of the six unknown coefficients $C_{i}$ with $i=\{1,6\}$ introduced below. The geometric constants are the length $L$, the width $B$, and the height $H$.

To this end it is convenient to introduce the following dimensionless parameter $\beta$ related to the length-scale parameter $c_{y}$ by the expression

$$
\beta=\sqrt{1+\frac{A}{I} c_{y}^{2}}=\sqrt{1+\left(\frac{c_{y}}{\rho}\right)^{2}} \geq 1,
$$

where $\rho=\sqrt{I / A}$ is the radius of gyration.
The solution of the sixth-order differential equation (20) is

$$
\begin{aligned}
v(x)= & \frac{c_{x}^{3}}{\beta^{4}} e^{\left(\beta / c_{x}\right) x} C_{1}+\frac{c_{x}^{3}}{\beta^{4}} e^{-\left(\beta / c_{x}\right) x} C_{2}+C_{3}+C_{4} x \\
& +C_{5} x^{2}+C_{6} x^{3}
\end{aligned}
$$

and bending moment (22) is given by

$$
M=2 E I \beta^{2} C_{5}+6 E I \beta^{2} C_{6} x .
$$

The six unknown coefficients are evaluated by means of the boundary conditions provided by relations (24) at $x=0$ and by relations (25) at $x=L$, where $\mathscr{M}=0$ and $\mathscr{F}=F$.

Accordingly the six unknown coefficients of the FUGM appearing in the expression of transverse displacement (27) and of bending moment (28) are given by

$$
\begin{aligned}
& C_{1}=\frac{F}{E I\left(1+e^{\left(L / c_{x}\right) \beta}\right) \beta}, \\
& C_{2}=-\frac{e^{\left(L / c_{x}\right) \beta} F}{E I\left(1+e^{\left(L / c_{x}\right) \beta}\right) \beta}, \\
& C_{3}=\frac{c_{x}^{3}\left(-1+e^{\left(L / c_{x}\right) \beta}\right) F}{E I\left(1+e^{\left(L / c_{x}\right) \beta}\right) \beta^{5}}, \\
& C_{4}=-\frac{c_{x}^{2} F}{E I \beta^{4}}, \\
& C_{5}=\frac{F L}{2 E I \beta^{2}}, \\
& C_{6}=-\frac{F}{6 E I \beta^{2}} .
\end{aligned}
$$

The lower and upper bounds $v_{0}$ and $v_{\infty}$ of the nanocantilever transverse displacement $v$ can then be evaluated by taking the limit of $v$ for $c_{x} \rightarrow 0$ and $c_{x} \rightarrow+\infty$, respectively, and are given by

$$
\begin{aligned}
v_{0} & =\frac{F L x^{2}}{2 E I}-\frac{F x^{3}}{6 E I}, \\
v_{\infty} & =\frac{F L x^{2}}{4 E I \beta^{2}} .
\end{aligned}
$$

Hence, the displacement $v$ of the FUGM belongs to the strip bounded by the functions $v_{0}$ and $v_{\infty}$. Note that the lower bound coincides with the classical (local) displacement $v_{0}$ of the considered nanocantilever and the upper bound $v_{\infty}$ depends on $\beta$, that is, the length-scale parameter $c_{y}$. Moreover, the nonlocal displacement $v$ tends to vanish for $\beta \rightarrow \infty$.

The bending moment (28) of the considered statically determined nanobeam reduces to its classical (local) counterpart $M=F(L-x)$ and the shear force is $T=-M^{(1)}=F$. A general theoretical motivation of this result is reported in [20]. 
Remark 2. The expression of the transverse displacement pertaining to the GM is obtained from (27) of the FUGM by setting $\beta=1$ (i.e., $c_{y}=0$ ) into the expressions of the six coefficients (29).

Let us now introduce the following dimensionless quantities:

$$
\begin{aligned}
\xi & =\frac{x}{L}, \\
\eta & =\frac{y}{L}, \\
b & =\frac{B}{L}, \\
h & =\frac{H}{L}, \\
\tau_{x} & =\frac{c_{x}}{L}, \\
\tau_{y} & =\frac{c_{y}}{L}, \\
v^{*} & =v \frac{E I}{F L^{3}} .
\end{aligned}
$$

Thus, the parameter $\beta$ (see (26)) can be rewritten as $\beta=$ $\sqrt{1+12\left(\tau_{y} / h\right)^{2}}$ and its plot is reported in Figure 1 in terms of the dimensionless ratio $\tau_{y} / h=c_{y} / H$.

For computations, we assume the following values of the dimensionless parameters: $\tau_{x} \in\{0,0.1,0.2,0.3,0.4,0.5\}$ and $\beta \in\{1,1.1,1.2,1.5,2\}$ which yields $\tau_{y} / h \in\{0,0.13,0.19,0.32$, $0.5\}$.

The transverse dimensionless deflection $v^{*}$ of the nanocantilever is plotted in Figure 2 for $\tau_{x}=0$ with $\beta=1$ and for $\tau_{x}=0.2$ with $\beta \in\{1,1.1,1.2,1.5,2\}$.

Note that the transverse dimensionless deflection $v^{*}$ obtained by the FUGM

(i) with $\tau_{x}=0$ and $\beta=1$ coincides with the classical (local) solution $v_{0}^{*}$;

(ii) with $\tau_{x} \neq 0$ and $\beta=1$ coincides with the one obtained by the GM with the corresponding $\tau_{x}$;

(iii) for a given $\tau_{x}$ and for increasing values of $\beta$ the nanocantilever becomes stiffer than the solution obtained by the GM.

The dimensionless upper bound $v_{0}^{*}$ of the transverse deflection is independent of the length-scale parameters $\left(\tau_{x}, \beta\right)$ and is reported in Figure 2 with the black dot-dashed line. The dimensionless lower bound $v_{\infty}^{*}$ depends on the parameter $\beta$ and is reported in Figure 2 with a coloured dotdashed line for the considered values of $\beta$. Hence, for a given $\beta$, the transverse dimensionless deflection $v^{*}$ of the FUGM belongs to the strip bounded by $v_{0}^{*}$ and by $v_{\infty}^{*}$, corresponding to the chosen $\beta$, for any value of the dimensionless lengthscale parameter $\tau_{x}$.

The transverse dimensionless deflection $v^{*}$ of the nanocantilever is reported in Figure 3 for $\tau_{x}=0$ and

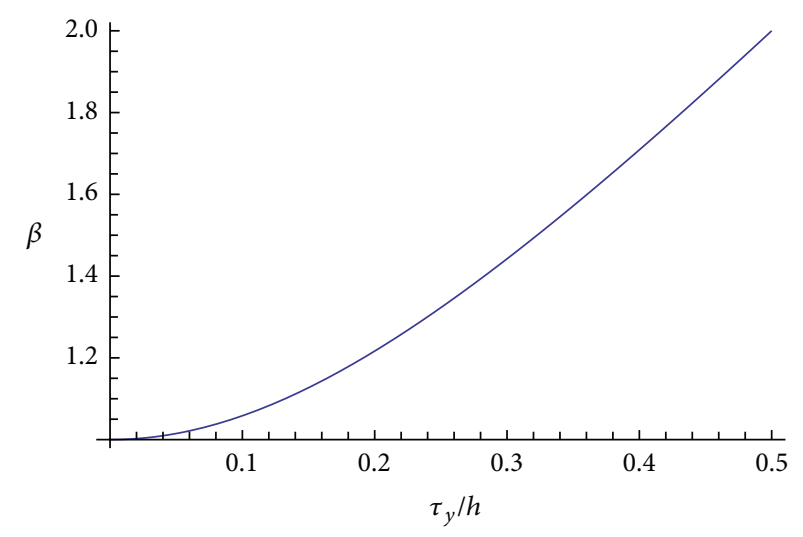

Figure 1: Parameter $\beta$ in terms of the dimensionless ratio $\tau_{y} / h=$ $c_{y} / H$.

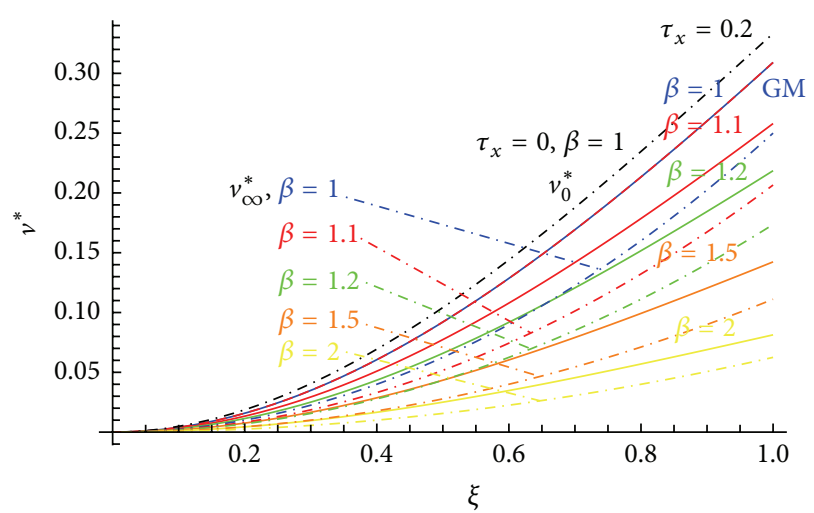

FIGURE 2: Transverse dimensionless deflection $v^{*}$ of the nanocantilever for $\tau_{x}=0$, with $\beta=1$, and for $\tau_{x}=0.2$, with $\beta \in\{1,1.1,1.2,1.5,2\}$, the dimensionless upper bound $v_{0}^{*}$, and the dimensionless lower bound $v_{\infty}^{*}$ depending on the parameter $\beta$.

$\beta \in\{1,1.1,1.2,1.5,2\}$. The vanishing of the length-scale parameter $\tau_{x}$ ensures that the GM provides the same solution of the classical (local) model. On the contrary, the FUGM induces a nonlocal behaviour due to the presence of the parameter $\beta$ as shown in Figure 3. As a consequence, the nanocantilever becomes stiffer than the local one for increasing values of the nonlocal parameter $\beta$.

The maximum dimensionless deflection $v^{*}(1)$ of the nanocantilever obtained by the FUGM is plotted in Figure 4 in terms of the dimensionless parameter $\beta$ for different values of $\tau_{x}$. The magnitude of $\beta$ ranges in the interval $[1,4], \tau_{x} \epsilon$ $\{0,0.1,0.2,0.3,0.4,0.5\}$, and all the plots have a horizontal asymptote $v^{*}=0$ for $\beta \rightarrow+\infty$; that is, $\tau_{y} \rightarrow+\infty$.

Denoting by $v_{G}^{*}(1)$ the maximum dimensionless deflection of the nanocantilever obtained by the GM, the comparison between $v^{*}(1)$ and $v_{G}^{*}(1)$ is reported in Table 1 where the variation $\Delta$ between the two maximum dimensionless deflections is evaluated according to formula $\Delta=\left[v^{*}(1)-\right.$ $\left.v_{G}^{*}(1)\right] / v_{G}^{*}(1)$.

The maximum dimensionless deflection in the classical (local) Euler-Bernoulli model is $v_{0}^{*}(1)=1 / 3$ and is recovered from the FUGM by setting $\tau_{x}=0$ and $\beta=1$; that is, $\tau_{y}=0$. 


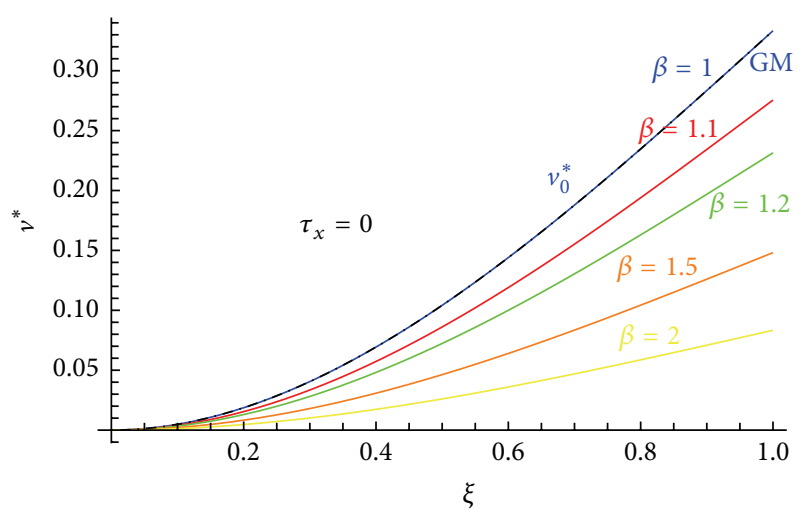

Figure 3: The transverse dimensionless deflection $v^{*}$ of the nanocantilever for $\tau_{x}=0$ and $\beta \in\{1,1.1,1.2,1.5,2\}$.

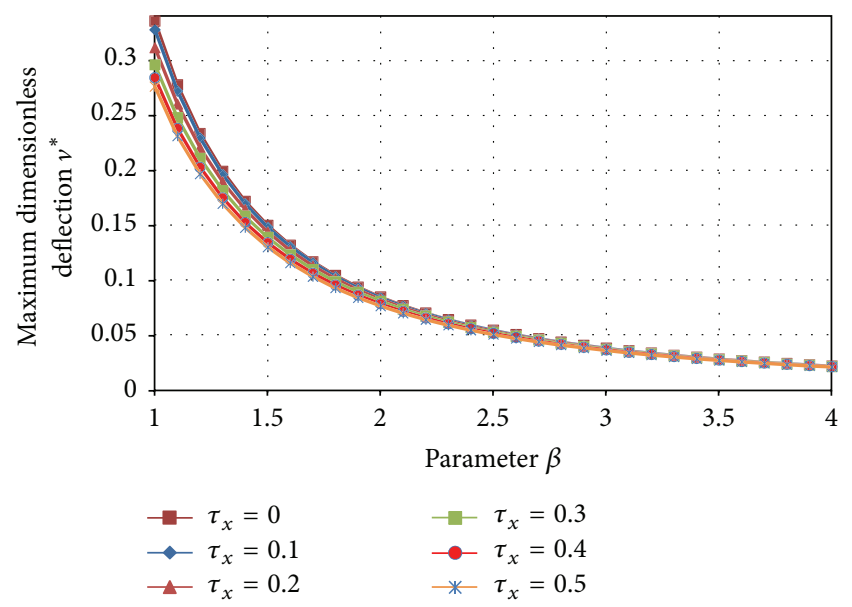

FIGURE 4: Maximum dimensionless deflection $v^{*}(1)$ of the nanocantilever in terms of the dimensionless parameter $\beta$ for $\tau_{x}$ ranging in the set $\{0,0.1,0.2,0.3,0.4,0.5\}$.

Table 1 clearly shows that the maximum dimensionless deflection evaluated by the FUGM quickly decreases for increasing $\beta$ with respect to the corresponding one of the GM. Analogously, for a given $\beta$, the FUGM becomes stiffer than the corresponding GM for increasing $\tau_{x}$.

\section{Conclusions}

A fully gradient nanobeam model (FUGM) is presented for the static bending of nanobeams based on a consistent thermodynamic approach. The governing equations and the related high-order boundary conditions are derived by using a variational formulation of the nonlocal model. The proposed theory can be specialized to recover the gradient elasticity beam model (GM).

Analytical solutions of the proposed nonlocal fully gradient nanobeam model in terms of transverse displacements are provided for nanocantilevers.

Present results are compared with the corresponding ones obtained using the gradient elasticity model to establish the validity of the present formulation.
TABLE 1: Comparison between the maximum dimensionless deflections $v^{*}(1)$ of the FUGM and $v_{G}^{*}(1)$ of the GM for different values of $\tau_{x}$ and $\beta$ for the considered nanocantilever. The variation is $\Delta=$ $\left[v^{*}(1)-v_{G}^{*}(1)\right] / v_{G}^{*}(1)$.

\begin{tabular}{|c|c|c|c|c|}
\hline$\tau_{x}$ & $v_{G}^{*}(1)$ & $\beta$ & $v^{*}(1)$ & $\Delta \%$ \\
\hline \multirow{5}{*}{0} & \multirow{5}{*}{0.333333} & 1.0 & 0.333333 & $0 \%$ \\
\hline & & 1.1 & 0.275482 & $-17.36 \%$ \\
\hline & & 1.2 & 0.231481 & $-30.56 \%$ \\
\hline & & 1.5 & 0.148148 & $-55.56 \%$ \\
\hline & & 2.0 & 0.0833333 & $-75.00 \%$ \\
\hline \multirow{5}{*}{0.1} & \multirow{5}{*}{0.325333} & 1.0 & 0.325333 & $0 \%$ \\
\hline & & 1.1 & 0.269894 & $-17.04 \%$ \\
\hline & & 1.2 & 0.227463 & $-30.08 \%$ \\
\hline & & 1.5 & 0.146436 & $-54.99 \%$ \\
\hline & & 2.0 & 0.0827708 & $-74.56 \%$ \\
\hline \multirow{5}{*}{0.2} & \multirow{5}{*}{0.309119} & 1.0 & 0.309119 & $0 \%$ \\
\hline & & 1.1 & 0.258015 & $-16.53 \%$ \\
\hline & & 1.2 & 0.21859 & $-29.29 \%$ \\
\hline & & 1.5 & 0.142352 & $-53.95 \%$ \\
\hline & & 2.0 & 0.0813333 & $-73.69 \%$ \\
\hline \multirow{5}{*}{0.3} & \multirow{5}{*}{0.293613} & 1.0 & 0.293613 & $0 \%$ \\
\hline & & 1.1 & 0.245869 & $-16.26 \%$ \\
\hline & & 1.2 & 0.208999 & $-28.82 \%$ \\
\hline & & 1.5 & 0.137386 & $-53.21 \%$ \\
\hline & & 2.0 & 0.0793915 & $-72.96 \%$ \\
\hline \multirow{5}{*}{0.4} & \multirow{5}{*}{0.281914} & 1.0 & 0.281914 & $0 \%$ \\
\hline & & 1.1 & 0.236127 & $-16.24 \%$ \\
\hline & & 1.2 & 0.200882 & $-28.74 \%$ \\
\hline & & 1.5 & 0.132625 & $-52.96 \%$ \\
\hline & & 2.0 & 0.0772798 & $-72.59 \%$ \\
\hline \multirow{5}{*}{0.5} & \multirow{5}{*}{0.273732} & 1.0 & 0.273732 & $0 \%$ \\
\hline & & 1.1 & 0.22899 & $-16.35 \%$ \\
\hline & & 1.2 & 0.194675 & $-28.88 \%$ \\
\hline & & 1.5 & 0.128565 & $-53.03 \%$ \\
\hline & & 2.0 & 0.0752398 & $-72.51 \%$ \\
\hline
\end{tabular}

The novelty of the present paper arises from the several points. Firstly, as stated above, a more general nonlocal nanobeam model that contains existing beam theories (classical and gradient elasticity theories) as special cases is proposed. Two nonlocal parameters are introduced in the FUGM in order to consider the nonlocal contributions in the beam axis direction and in the transverse direction.

Secondly, the following implications of technical interest are reported below.

(1) The proposed FUGM shows that an increasing of the nonlocal parameter associated with the strain gradient component in the transverse direction provides an increasing of the nanostructural stiffness so that the deflection of a nanobeam reduces with respect to the corresponding one of the local model; see Figures 2, 3 , and 4 . 
(2) The existing gradient elasticity model (GM) of EulerBernoulli nanobeams overestimates the displacement solution when compared with the new gradient elasticity model (FUGM) as reported in Figures 2-3.

(3) The proposed FUGM provides upper and lower bounds for displacements in terms of the parameter $\beta$. The upper bound is independent of the small-scale parameters while the lower bound depends on the nonlocal parameter $\beta$.

(4) The results given in Table 1 can be useful benchmarks for other researchers to compare their outcomes in the future.

\section{Conflict of Interests}

The authors declare that there is no conflict of interests regarding the publication of this paper.

\section{Acknowledgment}

The present study has been developed within the activities of the research program FARO 2012, Compagnia San Paolo, Polo delle Scienze e delle Tecnologie, University of Naples Federico II.

\section{References}

[1] J. Pei, F. Tian, and T. Thundat, "Glucose biosensor based on the microcantilever," Analytical Chemistry, vol. 76, no. 2, pp. 292297, 2004.

[2] M. Li, H. X. Tang, and M. L. Roukes, "Ultra-sensitive NEMSbased cantilevers for sensing, scanned probe and very highfrequency applications," Nature Nanotechnology, vol. 2, no. 2, pp. 114-120, 2007.

[3] Y. Fu, H. Du, W. Huang, S. Zhang, and M. Hu, “TiNi-based thin films in MEMS applications: a review," Sensors and Actuators, A: Physical, vol. 112, no. 2-3, pp. 395-408, 2004.

[4] Z. Lee, C. Ophus, L. M. Fischer et al., "Metallic NEMS components fabricated from nanocomposite Al-Mo films," Nanotechnology, vol. 17, no. 12, article 3063, 2006.

[5] A. Witvrouw and A. Mehta, "The use of functionally graded poly-SiGe layers for MEMS applications," Materials Science Forum, vol. 492-493, pp. 255-260, 2005.

[6] W. J. Poole, M. F. Ashby, and N. A. Fleck, "Micro-hardness of annealed and work-hardened copper polycrystals," Scripta Materialia, vol. 34, no. 4, pp. 559-564, 1996.

[7] E. M. Abdel-Rahman, M. I. Younis, and A. H. Nayfeh, "Characterization of the mechanical behavior of an electrically actuated microbeam," Journal of Micromechanics and Microengineering, vol. 12, no. 6, pp. 759-766, 2002.

[8] S. Cuenot, C. Frétigny, S. Demoustier-Champagne, and B. Nysten, "Surface tension effect on the mechanical properties of nanomaterials measured by atomic force microscopy," Physical Review B, vol. 69, no. 16, Article ID 165410, 2004.

[9] C. M. Craciunescu and M. Wuttig, "New ferromagnetic and functionally graded shape memory alloys," Journal of Optoelectronics and Advanced Materials, vol. 5, no. 1, pp. 139-146, 2003.

[10] H. J. Xiang and J. Yang, "Free and forced vibration of a laminated FGM Timoshenko beam of variable thickness under heat conduction," Composites-Part B: Engineering, vol. 39, no. 2, pp. 292-303, 2008.

[11] M. Simsek and J. N. Reddy, "Bending and vibration of functionally graded microbeams using a new higher order beam theory and the modified couple stress theory," International Journal of Engineering Science, vol. 64, pp. 37-53, 2013.

[12] D. Qian, G. J. Wagner, W. K. Liu, M. Yu, and R. S. Ruoff, "Mechanics of carbon nanotubes," Applied Mechanics Reviews, vol. 55, no. 6, p. 495, 2002.

[13] R. Larsson and K. Samadikhah, "Atomistic continuum modeling of graphene membranes," Computational Materials Science, vol. 50, no. 5, pp. 1744-1753, 2011.

[14] Y. Chandra, F. Scarpa, R. Chowdhury, S. Adhikari, and J. Sienz, "Multiscale hybrid atomistic-FE approach for the nonlinear tensile behaviour of graphene nanocomposites," Composites Part A: Applied Science and Manufacturing, vol. 46, no. 1, pp. 147-153, 2013.

[15] R. Barretta, F. M. de Sciarra, and M. Diaco, "Small-scale effects in nanorods," Acta Mechanica, vol. 225, no. 7, pp. 1945-1953, 2014.

[16] F. M. de Sciarra and R. Barretta, "A new nonlocal bending model for Euler-Bernoulli nanobeams," Mechanics Research Communications, vol. 62, no. 1, pp. 25-30, 2014.

[17] A. C. Eringen, "On differential equations of nonlocal elasticity and solutions of screw dislocation and surface waves," Journal of Applied Physics, vol. 54, no. 9, pp. 4703-4710, 1983.

[18] A. C. Eringen, Nonlocal Continuum Field Theories, Springer, New York, NY, USA, 2002.

[19] K. A. Lazopoulos and A. K. Lazopoulos, "Bending and buckling of thin strain gradient elastic beams," European Journal of Mechanics, A/Solids, vol. 29, no. 5, pp. 837-843, 2010.

[20] R. Barretta and F. Marotti de Sciarra, "Analogies between nonlocal and local Bernoulli-Euler nanobeams," Archive of Applied Mechanics, vol. 85, no. 1, pp. 89-99, 2014.

[21] F. M. de Sciarra, "Finite element modelling of nonlocal beams," Physica E: Low-Dimensional Systems and Nanostructures, vol. 59, pp. 144-149, 2014.

[22] R. Barretta, L. Feo, and R. Luciano, "Torsion of functionally graded nonlocal viscoelastic circular nanobeams," Composites-Part B: Engineering, vol. 72, pp. 217-222, 2015.

[23] R. Barretta, L. Feo, R. Luciano, and F. M. de Sciarra, "Variational formulations for functionally graded nonlocal Bernoulli-Euler nanobeams," Composite Structures, vol. 129, pp. 80-89, 2015.

[24] R. Barretta, L. Feo, R. Luciano, and F. M. de Sciarra, "A gradient Eringen model for functionally graded nanorods," Composite Structures, vol. 131, pp. 1124-1131, 2015.

[25] R. D. Mindlin and H. F. Tiersten, "Effects of couple-stresses in linear elasticity," Archive for Rational Mechanics and Analysis, vol. 11, pp. 415-448, 1962.

[26] A. R. Hadjesfandiari and G. F. Dargush, "Couple stress theory for solids," International Journal of Solids and Structures, vol. 48, no. 18, pp. 2496-2510, 2011.

[27] F. Yang, A. C. M. Chong, D. C. C. Lam, and P. Tong, "Couple stress based strain gradient theory for elasticity," International Journal of Solids and Structures, vol. 39, no. 10, pp. 2731-2743, 2002.

[28] M. Asghari, M. H. Kahrobaiyan, M. Rahaeifard, and M. T. Ahmadian, "Investigation of the size effects in Timoshenko beams based on the couple stress theory," Archive of Applied Mechanics, vol. 81, no. 7, pp. 863-874, 2011. 
[29] H. M. Ma, X.-L. Gao, and J. N. Reddy, "A microstructuredependent Timoshenko beam model based on a modified couple stress theory," Journal of the Mechanics and Physics of Solids, vol. 56, no. 12, pp. 3379-3391, 2008.

[30] E. C. Aifantis, "On the microstructural origin of certain inelastic models," Journal of Engineering Materials and Technology, Transactions of the ASME, vol. 106, no. 4, pp. 326-330, 1984.

[31] E. C. Aifantis, "The physics of plastic deformation," International Journal of Plasticity, vol. 3, no. 3, pp. 211-247, 1987.

[32] R. H. J. Peerlings, R. de Borst, W. A. M. Brekelmans, and J. H. P. de Vree, "Gradient enhanced damage for quasi-brittle materials," International Journal for Numerical Methods in Engineering, vol. 39, no. 19, pp. 3391-3403, 1996.

[33] F. M. de Sciarra, "Variational formulations, convergence and stability properties in nonlocal elastoplasticity," International Journal of Solids and Structures, vol. 45, no. 7-8, pp. 2322-2354, 2008.

[34] E. C. Aifantis, "On the role of gradients in the localization of deformation and fracture," International Journal of Engineering Science, vol. 30, no. 10, pp. 1279-1299, 1992.

[35] C. Q. Ru and E. C. Aifantis, "A simple approach to solve boundary value problems in gradient elasticity," Acta Mechanica, vol. 101, no. 1-4, pp. 59-68, 1993.

[36] L. T. Tenek and E. C. Aifantis, "A two-dimensional finite element implementation of a special form of gradient elasticity," Computer Modeling in Engineering \& Sciences, vol. 3, no. 6, pp. 731-741, 2002.

[37] E. C. Aifantis, "Update on a class of gradient theories," Mechanics of Materials, vol. 35, no. 3-6, pp. 259-280, 2003.

[38] H. Askes, I. Morata, and E. C. Aifantis, "Finite element analysis with staggered gradient elasticity," Computers and Structures, vol. 86, no. 11-12, pp. 1266-1279, 2008.

[39] H. Askes and I. M. Gitman, "Non-singular stresses in gradient elasticity at bi-material interface with transverse crack," International Journal of Fracture, vol. 156, no. 2, pp. 217-222, 2009.

[40] H. Askes and E. C. Aifantis, "Gradient elasticity in statics and dynamics: an overview of formulations, length scale identification procedures, finite element implementations and new results," International Journal of Solids and Structures, vol. 48, no. 13, pp. 1962-1990, 2011.

[41] R. Luciano and J. R. Willis, "Boundary-layer corrections for stress and strain fields in randomly heterogeneous materials," Journal of the Mechanics and Physics of Solids, vol. 51, no. 6, pp. 1075-1088, 2003.

[42] R. Luciano and J. R. Willis, "Hashin-Shtrikman based FE analysis of the elastic behaviour of finite random composite bodies," International Journal of Fracture, vol. 137, no. 1-4, pp. 261-273, 2006.

[43] K. Y. Xu, K. A. Alnefaie, N. H. Abu-Hamdeh, K. H. Almitani, and E. C. Aifantis, "Free transverse vibrations of a doublewalled carbon nanotube: gradient and internal inertia effects," Acta Mechanica Solida Sinica, vol. 27, no. 4, pp. 345-352, 2014.

[44] J. Lemaitre and L. Chaboche, Mechanics of Solid Materials, Cambridge University Press, Cambridge, UK, 1994.

[45] F. Marotti de Sciarra, "Hardening plasticity with nonlocal strain damage," International Journal of Plasticity, vol. 34, pp. 114-138, 2012.

[46] F. M. de Sciarra, "On non-local and non-homogeneous elastic continua," International Journal of Solids and Structures, vol. 46, no. 3-4, pp. 651-676, 2009.
[47] D. G. B. Edelen and N. Laws, "On the thermodynamics of systems with nonlocality," Archive for Rational Mechanics and Analysis, vol. 43, pp. 24-35, 1971.

[48] F. Marotti de Sciarra, "A general theory for nonlocal softening plasticity of integral-type," International Journal of Plasticity, vol. 24, no. 8, pp. 1411-1439, 2008. 


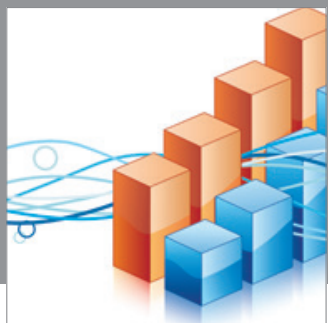

Advances in

Operations Research

mansans

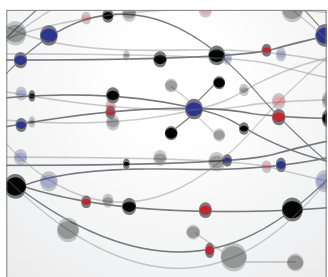

The Scientific World Journal
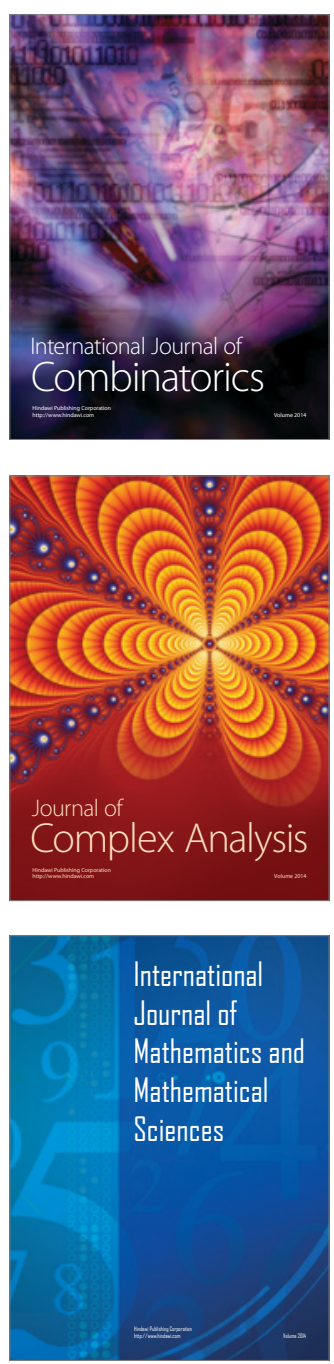
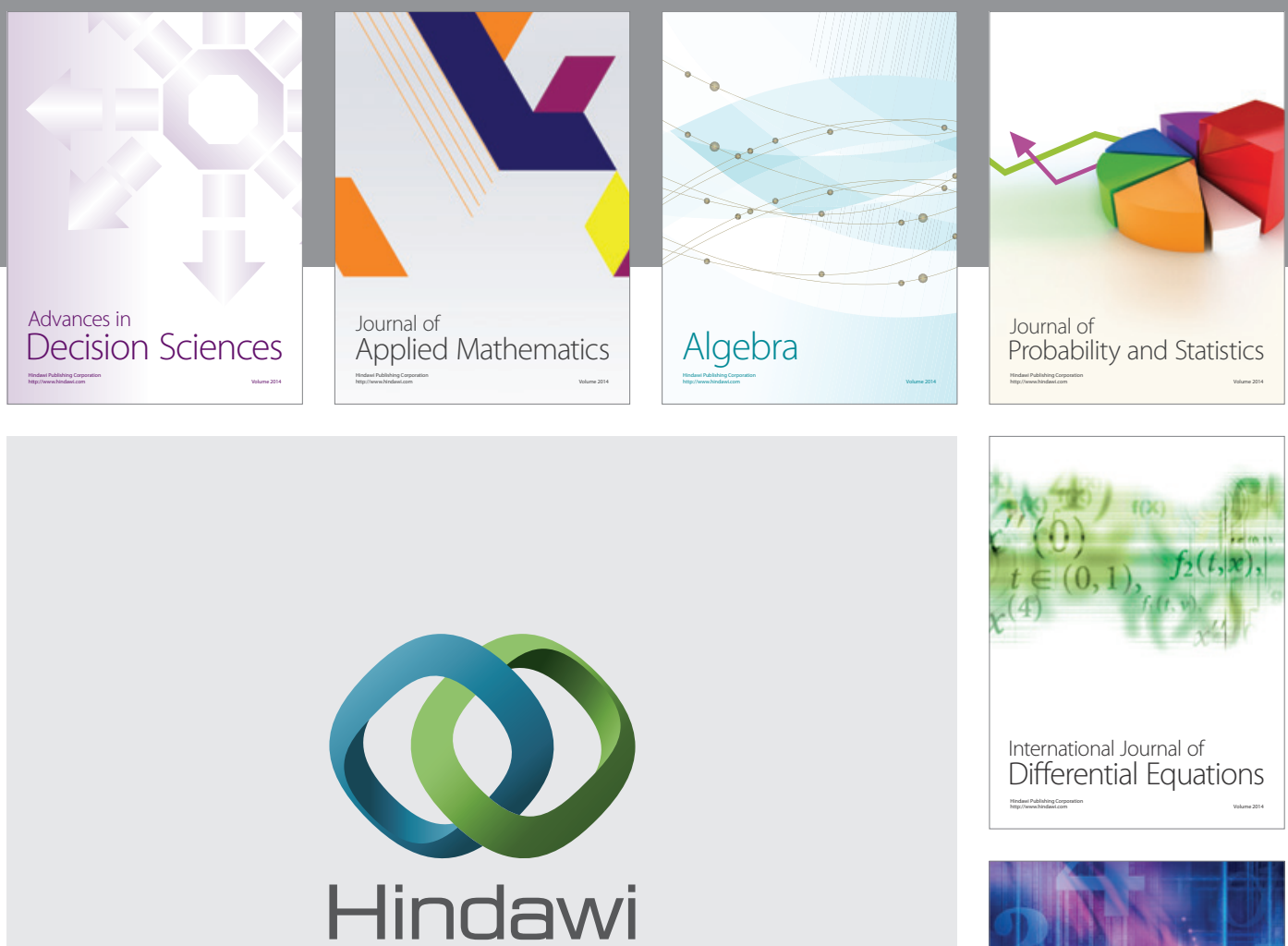

Submit your manuscripts at http://www.hindawi.com
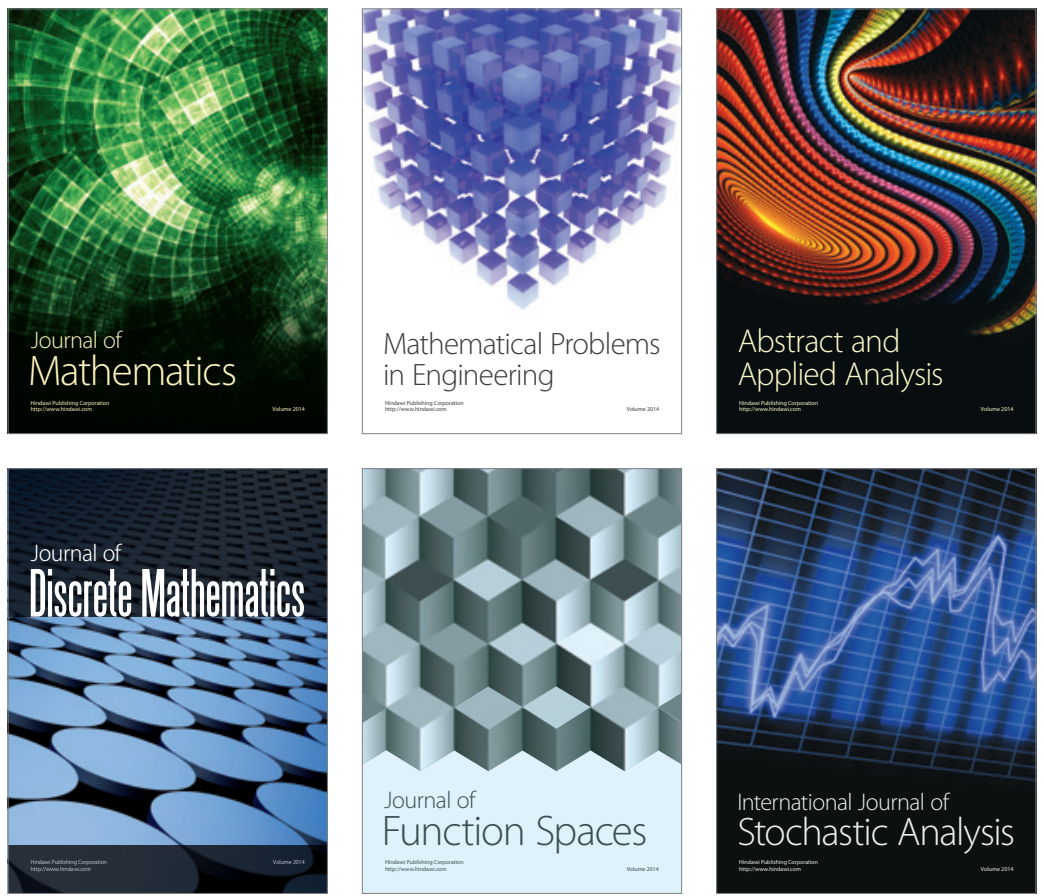

Journal of

Function Spaces

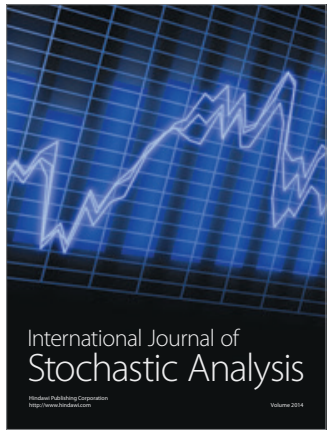

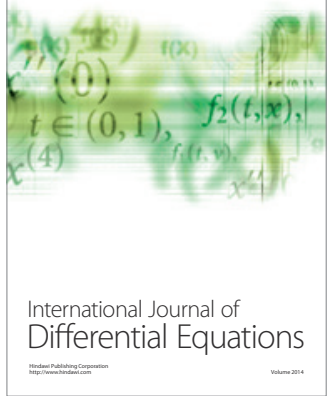
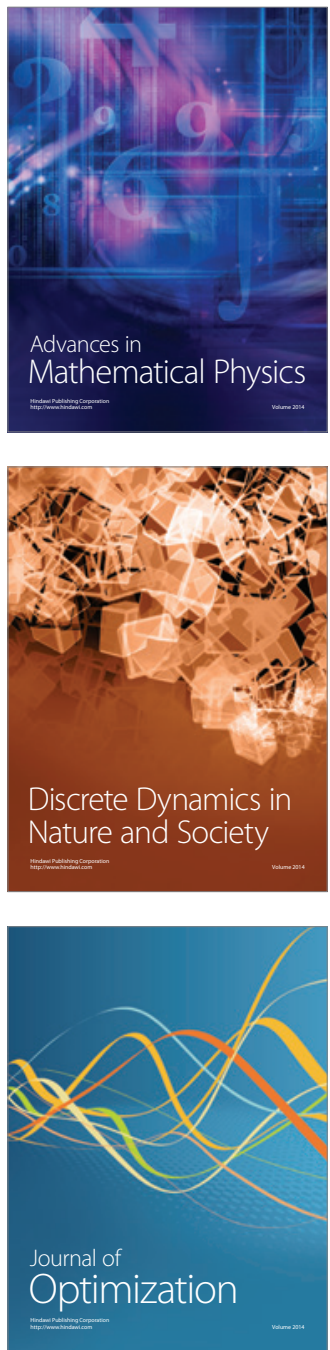